\title{
Atlas of Common Pain Syndromes, 3rd Edition
}

\author{
Steven D. Waldman. Elsevier Saunders, Philadelphia USA, 2012, \\ US \$119, 396 pages. ISBN 978-1-4377-3792-9
}

\author{
Alexander J. Clark, MD
}

Received: 26 October 2011/ Accepted: 31 October 2011/Published online: 9 November 2011

(C) Canadian Anesthesiologists' Society 2011

Waldman's Atlas of Common Pain Syndromes, $3^{\text {rd }}$ Edition has been enhanced by the addition of 18 chapters, improved visuals with more ultrasound and radiographic images, and an expanded physical examination section. The author acknowledges that the motivation for this edition is "to help practitioners move beyond the constraints of our common diagnostic constructs" and "to focus on diagnosis rather than treatment". On-line access to the book is also available through Elsevier's ExpertConsult (www.expertconsult.com).

Each of the 124 chapters details a specific pain condition and provides a step-by-step approach which progresses from signs and symptoms through physical findings, laboratory and radiographic testing, treatment options, complications and pitfalls, and clinical pearls. Diagnostic codes (ICD-9 and -10) are provided for each condition, and a brief list of suggested readings is provided for each chapter. The extensive breadth of topics is separated into 16 sections, including Headache Pain Syndromes, Shoulder Pain Syndromes, Chest Wall Pain Syndromes, Hip and Lower Extremity Pain Syndromes, Ankle Pain Syndromes, etc. Each section comprises four to 15 chapters that detail specific pain conditions relevant to the particular body area. The entire list of conditions is comprehensive and covers most common pain syndromes.

This author describes each pain condition clearly and in simple terms with details of the commonly observed signs and symptoms, the appropriate diagnostic testing that can be utilized, and a differential diagnosis for the pain syndrome. Images are often provided as examples of alternative

A. J. Clark, MD ( $\bowtie)$

Dalhousie University, Halifax, NS, Canada

e-mail: john.clark@cdha.nshealth.ca diagnostic possibilities. The use of visual aids is especially helpful and the chapters are short and to the point.

The suggested treatment for each of the pain conditions is typically laid out in a hierarchical manner with no indication of "best evidence" for the condition. A reader can only assume that the treatment approach is that of the author. Treatment recommendations are often generic in nature, e.g., use of NSAIDs, COXIBs, physical therapy, and injections, rather than specific regarding medications and/or modalities.

Frequently, injection techniques are advocated as appropriate therapy and are described in detail, giving the reader the impression that the technique might be an important part of the treatment. In some instances, the recommendation to perform injections is not supported by current guidelines or by "evidence-based" medicine. Examples include the use of stellate ganglion blocks for reflex sympathetic dystrophy of the face, intercostal nerve blocks for truncal diabetic neuropathy, epidural injections for post-herpetic neuralgia, and injections for acute pancreatitis. The reader should bear in mind that the evidence for the use of injections for many pain conditions is weak and sometimes extremely limited. It should also be remembered that injection therapies do have side effects and complications that can be significant. Waldman does detail possible complications of nerve blocks in most chapters where they are suggested as part of treatment.

The "Clinical Pearl" section at the end of each chapter is a useful summary of the pain condition, issues pertaining to the condition, as well as other conditions to be considered in the differential diagnosis, investigations, treatment, complications, and pitfalls.

Some outdated terms are used in the book, e.g., reflex sympathetic dystrophy, and other terms are inappropriate, e.g., fibromyalgia of the cervical musculature. 
Overall, this is a useful book for any physician who cares for patients with chronic pain as it provides a logical decision-making process towards diagnosis together with many excellent pictures and visuals of the relevant anatomy. The major limitation of the book is the lack of an "evidence-based" approach to treatment and the overreliance on injections as part of care.

Competing interests None declared. 